\title{
A synergistic core for human brain evolution and cognition
}

\author{
Andrea I. Luppi ${ }^{\mathrm{a}, \mathrm{b} *}$, Pedro A.M. Mediano ${ }^{\mathrm{c}}$, Fernando E. Rosas ${ }^{\mathrm{d}, \mathrm{e}, \mathrm{f}}$, Negin Holland $^{\mathrm{a}}$, Tim D.
} Fryer $^{\mathrm{a}, \mathrm{g}}$, John T. O’Brien ${ }^{\mathrm{h}, \mathrm{i}}$, James B. Rowe ${ }^{\mathrm{a}, \mathrm{i}, \mathrm{j}}$, David K. Menon ${ }^{\mathrm{a}, \mathrm{g}}$, Daniel Bor ${ }^{\mathrm{c}}$, \& Emmanuel

A. Stamatakis ${ }^{b}$

\author{
aDepartment of Clinical Neurosciences, University of Cambridge \\ ${ }^{b}$ University Division of Anaesthesia, School of Clinical Medicine, University of Cambridge \\ ${ }^{c}$ Department of Psychology, University of Cambridge \\ ${ }^{\mathrm{d}}$ Center for Psychedelic Research, Department of Brain Science, Imperial College London \\ ${ }^{\mathrm{e}} \mathrm{Center}$ for Complexity Science, Imperial College London \\ fData Science Institute, Imperial College London \\ ${ }^{\mathrm{g}}$ Wolfson Brain Imaging Centre, University of Cambridge \\ ${ }^{\mathrm{h}}$ Department of Psychiatry, University of Cambridge \\ ${ }^{\text {i }}$ Cambridge University Hospitals NHS Foundation Trust, Cambridge, UK \\ ${ }^{\mathrm{j}}$ MRC Cognition and Brain Sciences Unit, University of Cambridge \\ *Corresponding author:al857@ cam.ac.uk
}

\begin{abstract}
A fundamental question in neuroscience is how brain organisation gives rise to humans' unique cognitive abilities. Although complex cognition is widely assumed to rely on frontal and parietal brain regions, the underlying mechanisms remain elusive: current approaches are unable to disentangle different forms of information processing in the brain. Here, we introduce a powerful framework to identify synergistic and redundant contributions to neural information processing and cognition. Leveraging multimodal data including functional MRI, PET, cytoarchitectonics and genetics, we reveal that synergistic interactions are the fundamental drivers of complex human cognition. Whereas redundant information dominates sensorimotor areas, synergistic activity is closely associated with the brain's prefrontal-parietal and default networks; furthermore, meta-analytic results demonstrate a close relationship between highlevel cognitive tasks and synergistic information. From an evolutionary perspective, the human brain exhibits higher prevalence of synergistic information than non-human primates. At the macroscale, we demonstrate that high-synergy regions underwent the highest degree of evolutionary cortical expansion. At the microscale, human-accelerated genes promote synergistic interactions by enhancing synaptic transmission. These convergent results provide critical insights that synergistic neural interactions underlie the evolution and functioning of humans' sophisticated cognitive abilities, and demonstrate the power of our widely applicable information decomposition framework.
\end{abstract}


Synergistic and redundant interactions identify brain networks with distinct neurocognitive profiles

In theoretical and cognitive neuroscience, considering the human brain as a distributed information-processing system has proven to be a powerful framework to understand the neural basis of cognition 1. Crucially, a deeper understanding of any information-processing architecture calls for a more nuanced account of the information that is being processed.

As an example, let us consider humans' two main sources of information about the world: the eyes. The information that we still have when we close either eye is called "redundant information" - because it is information that can be conveyed by either source (for instance, information about colour is largely redundant between the two eyes). Redundancy provides robustness: we can still see with one eye closed. However, closing one eye also deprives us of stereoscopic information about depth. This information does not come from either eye alone: ones needs both, in order to perceive the third dimension. This is called the "synergistic information" between two sources - the extra advantage that we derive from combining them, which makes them complementary ${ }^{2,3}$.

Thus, in addition to their own unique information, when multiple sources are considered together their information contribution can be identified as synergistic (only available when both sources are considered together) or redundant (available from either source independently). Every information-processing system — including the human brain — needs to strike a balance between these mutually exclusive kinds of information, and the advantages they provide: robustness and integration, respectively ${ }^{4-7}$. Being fundamentally different, synergistic and redundant information cannot be adequately captured by traditional measures of macroscale information exchange ("functional connectivity") in the human brain, which instead simply quantify the similarity between regional activity ${ }^{2,8}$.

Here, we reveal the distinct contributions of synergistic and redundant interactions to human cognition, and we delineate their large-scale organisation in the human brain. To this end, we leveraged the partial information decomposition (PID) framework ${ }^{2,3,9}$ to quantify synergistic and redundant interactions between brain regions (Figure 1A,B), obtained from resting-state functional MRI data from 100 Human Connectome Project subjects (Methods). We ranked each brain region separately in terms of how synergistic and redundant its interactions with other brain regions are; the difference between these ranks (synergy minus redundancy) 
bioRxiv preprint doi: https://doi.org/10.1101/2020.09.22.308981; this version posted September 22, 2020. The copyright holder for this preprint (which was not certified by peer review) is the author/funder, who has granted bioRxiv a license to display the preprint in perpetuity. It is made available under aCC-BY 4.0 International license.

(B) Synergy
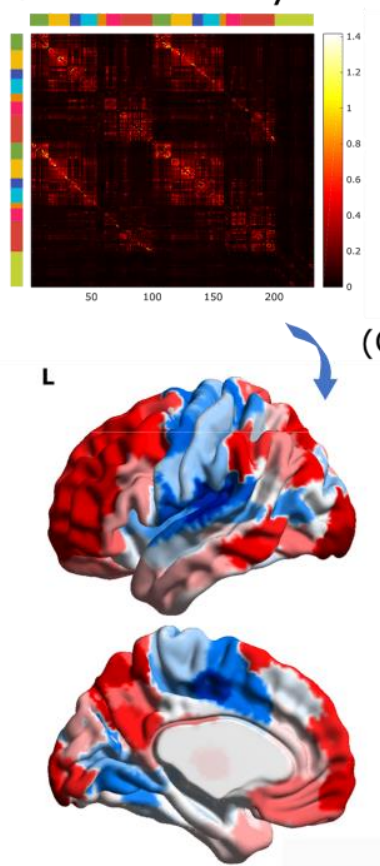

Synergy minus Redundancy rank gradient

(C)
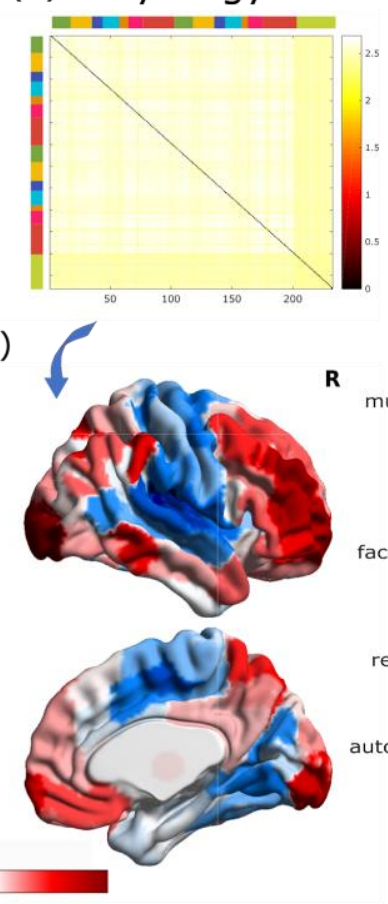

$\mathbf{R}$

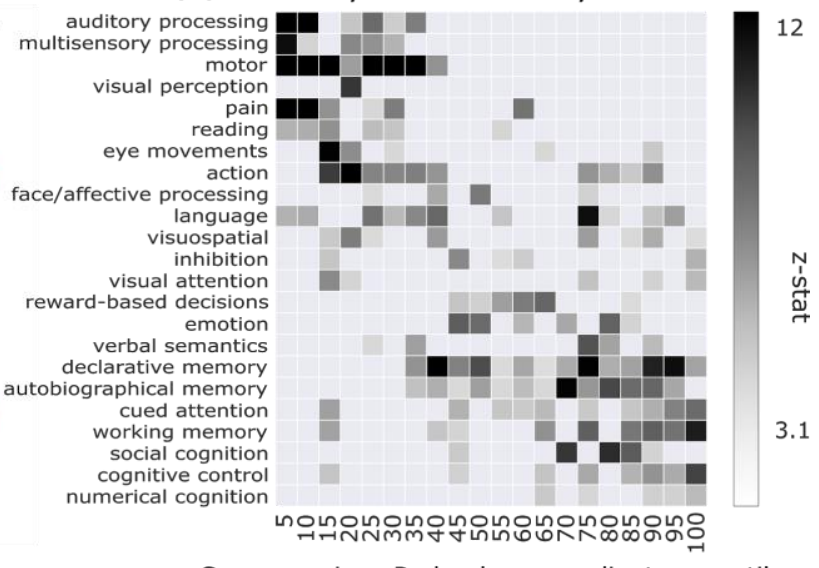

Figure 1. Synergistic and redundant networks exhibit distinct anatomical and cognitive profiles. Groupaverage matrices of redundant (A) and synergistic (B) interactions between regions of the 232-ROI augmented Schaefer atlas. (C) Brain surface projections of regional redundancy-to-synergy gradient scores, obtained as the difference between each region's rank in terms of synergy and in terms of redundancy; positive scores (red) indicate a bias towards synergy, and negative scores (blue) a bias towards redundancy. (D) Matrix of redundancyto-synergy gradient scores (synergy minus redundancy ranks) for each connection between brain regions. (E) Results of the NeuroSynth term-based meta-analysis, relating the distribution of redundancy-to-synergy gradient across the brain (discretised in 5\% increments) to a gradient of cognitive domains, from lower-level sensorimotor processing to higher-level cognitive tasks. These results are robust to the use of different parcellations (corticalonly, having lower or higher number of nodes, and obtained from anatomical rather than functional considerations; Figure S1A-C) and are also replicated without deconvolving the hemodynamic response function from the functional data (Figure S1D).

Our results demonstrate that traditional FC mostly captures redundant, rather than synergistic, information exchange in the human brain (Figure S2). Furthermore, they clearly show that redundant and synergistic interactions delineate networks with distinct neuroanatomical profiles (Figure 1A-D). In terms of Von Economo's cytoarchitectonic classification 10, redundant interactions are especially prominent in primary sensory, primary motor and insular cortices (Figure S3), corresponding to the brain's somatomotor and salience subnetworks 
91

92

93

94

95

96

97

98

(Figure S4). In contrast, regions with higher relative importance for synergy predominate in higher-order association cortex, and are affiliated with the default mode (DMN) and frontoparietal executive control (FPN) subnetworks ${ }^{11}$ (Figures S3-4).

It is noteworthy that synergy, which quantifies the extra information gained by integrating multiple sources ${ }^{3,12}$ is most prevalent in regions belonging to the DMN and FPN. Functionally, these regions are recruited by complex tasks that rely on multimodal information, decoupled from immediate sensorimotor contingencies ${ }^{13,14}$; anatomically, they receive multimodal inputs from across the brain ${ }^{15}$. Therefore, it has been speculated that these networks are devoted to the integration of information ${ }^{13,15}$. Our findings about regional prevalence of synergy in DMN and FPN provide formal information-theoretic evidence to confirm this long-standing hypothesis. Furthermore, by considering a synergy-redundancy gradient in terms of connections instead of regions, we show that the most synergy-dominated connections correspond to links between DMN/FPN and other subnetworks, whereas redundancydominated connections tend to occur within each subnetwork (Figure 1C).

The distinct cytoarchitectonic profiles and subnetwork affiliations further suggest that redundant and synergistic interactions may be involved with radically different cognitive domains. To empirically validate this hypothesis, we performed a term-based meta-analysis using NeuroSynth. The redundancy-to-synergy gradient identified in terms of regional rank differences was related to 24 terms pertaining to higher cognitive functions (e.g. attention, working memory, social and numerical cognition) and lower sensorimotor functions (such as eye movement, motion, visual and auditory perception) adopted by previous studies ${ }^{13,16}$.

Supporting the inference from neuroanatomy to cognition, our results reveal that the regional gradient from redundancy to synergy corresponds to a gradient from lower to higher cognitive functions. Specifically, high-redundancy regions loaded strongly onto auditory, visual and multisensory processing and motion. In contrast, high-synergy regions had the strongest loadings onto social and numerical cognition, working memory and cognitive control (Figure $1 \mathrm{E})$. 
Network organisation of synergy and redundancy support their distinct informationprocessing roles

122

Sensorimotor and higher-order cognitive functions impose distinct and opposite demands on cognitive architectures: specialised sensory processing benefits from segregation into modules, whereas integration of information demands high levels of interconnectedness ${ }^{5,17}$. Contrasting the properties of the networks delineated by synergistic and redundant interactions reveals how the human brain resolves this tension.

Across individuals, the network of synergistic interactions is more highly interconnected and globally efficient than the network of redundancy (Synergy: M=2.54, SD=0.06; Redundancy: $\mathrm{M}=0.14, \mathrm{SD}=0.04 ; \mathrm{t}(99)=-330.04, \mathrm{p}<0.001$, Hedge's $g=-46.67)$ (Figure 2A). In contrast, redundant interactions delineate a network characterised by a highly modular structure, which is virtually absent in synergistic networks (Synergy: $\mathrm{M}=0.005, \mathrm{SD}=0.001$; Redundancy: $\mathrm{M}=0.29, \mathrm{SD}=0.06 ; \mathrm{t}(99)=51.74, \mathrm{p}<0.001$, Hedge's $g=7.25$ ) (Figure 2B). Thus, synergistic and redundant interactions exhibit distinct network organisation, supporting integrated and segregated processing, respectively - as demanded by the cognitive functions they support.

It is also known that only a subset of regions are directly connected by white matter tracts ${ }^{18}$; therefore, we reasoned that the more an organism's survival depends on information exchange between regions $\mathrm{X}$ and $\mathrm{Y}$, the more one should expect $\mathrm{X}$ and $\mathrm{Y}$ to be directly connected. Thus, direct physical connections in the brain reveal where the need for robust communication is highest. Consequently, if redundant interdependencies are representative of robust information exchange, they should be co-located with underlying direct anatomical connections - as quantified using diffusion-weighted imaging (DWI). Our results support this hypothesis: across subjects, the number of white matter streamlines was significantly more correlated with redundant $(\mathrm{M}=0.16, \mathrm{SD}=0.028)$ than synergistic interactions between regions $(\mathrm{M}=0.025$, $\mathrm{SD}=0.015 ; \mathrm{t}(99)=39.85, \mathrm{p}<0.001$, Hedge's $g=6.29$ ) (Figure 2C,D). These results are replicated using alternative network measures and parcellations (Figures S5-7 and Supplementary Tables 1-3).

Thus, whereas synergistic interactions are poised to facilitate high-level cognition through global integration, redundant interactions demarcate a structural-functional backbone in the human brain, ensuring robust sensorimotor input-output channels - both critical functions for successful information processing. 
(A)

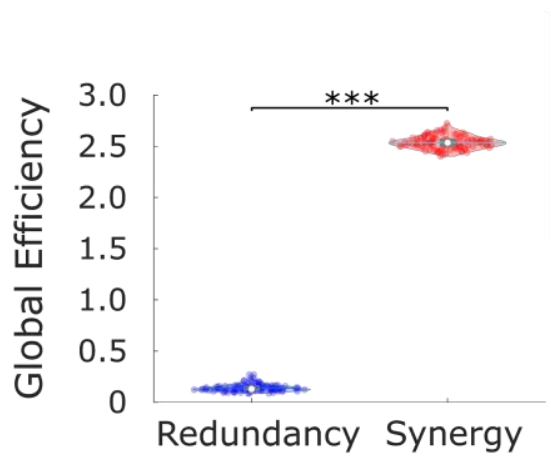

(B)

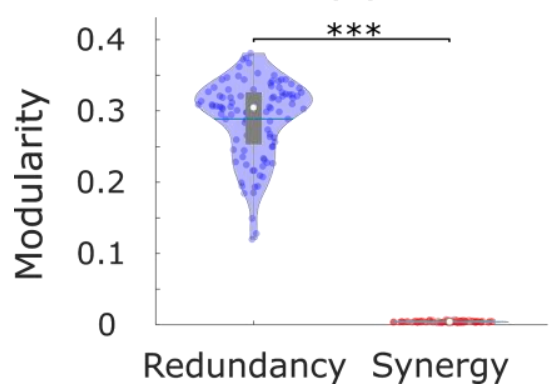

(C)

\section{Structural Connectivity}

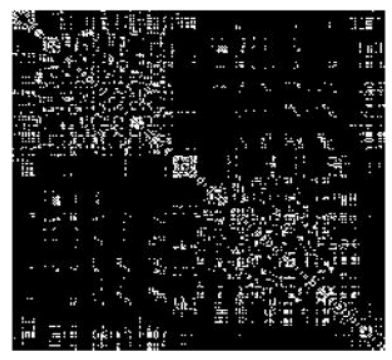

(D)

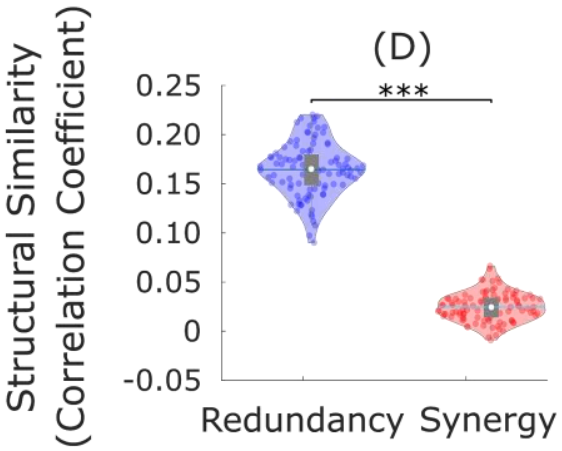

Figure 2. Synergy is integrated, redundancy is segregated and supported by anatomical connections. (A) The network organisation of synergistic interactions exhibits significantly higher integrative capacity (global efficiency) than redundant interactions. (B) The network organisation of redundant interactions exhibits significantly higher segregation (modularity) than synergistic interactions. (C) Structural connectivity of each subject was estimated from diffusion MRI, measured as the number of white matter tracts between regions of the 232-ROI augmented Schaefer atlas, and Spearman correlation coefficient was used to assess the similarity of redundancy and synergy matrices with structural connectivity, after thresholding to ensure equal numbers of connections. (D) Networks of redundant interactions are significantly more correlated with underlying structural connectivity than synergistic interactions. Violin plots represent the distribution of values across $100 \mathrm{HCP}$ subjects (colored circles). White circle: mean; blue line: median; grey box: interquartile range; *** $\mathrm{p}<0.001$.

High-synergy brain regions are selectively potentiated by human evolution

The association between synergistic information processing and higher cognitive functions, raises the intriguing possibility that the human brain may enable humans' uniquely sophisticated cognitive capacities in virtue of its highly synergistic nature. We pursued this hypothesis through three convergent approaches.

168 First, we show that the human brain is especially successful at leveraging synergistic information, compared with the brains of non-human primates. Synergistic interactions account

170 for a higher proportion of total information exchange in the human brain than in the macaque 171 (Macaca mulatta); whereas the two species' brains are equal in terms of proportion of total 172 information exchange accounted for by redundancy (Synergy: Human $\mathrm{M}=0.478, \mathrm{SD}=0.003$; 
173 Macaque $\mathrm{M}=0.466, \mathrm{SD}=0.005 ; \mathrm{t}(117)=14.24, \mathrm{p}<0.001$, Hedge's $\mathrm{g}=3.54$; Figure 3A;

174 Redundancy: Human $\mathrm{M}=0.012, \mathrm{SD}=0.005$; Macaque $\mathrm{M}=0.011, \mathrm{SD}=0.005 ; \mathrm{t}(117)=0.90$, $175 \mathrm{p}=0.372$, Hedge's g=0.22; Figure 3B).

176 The patterns of synergy and redundancy in the macaque brain broadly resemble those observed

177 in humans (Figure S8 and Supplementary Table 7), demonstrating their evolutionary stability 178 - including the expected high redundancy in sensorimotor regions (Figure 3C). However, 179 redundancy is more prevalent than synergy in the prefrontal cortex (PFC) of macaques, despite 180 PFC being among the most synergy-dominated cortices in humans (Figure 3C). Intriguingly, 181 prefrontal cortex underwent substantial cortical expansion in the course of human evolution ${ }^{19}$.

182 These findings suggest that the high synergy observed in human brains may be a specific outcome of evolutionary cortical expansion. To explore this hypothesis, we analysed cortical morphometry data from in vivo structural MRI, comparing humans and one of the closest evolutionary relatives of Homo sapiens: chimpanzees (Pan troglodytes) ${ }^{20}$. Supporting our hypothesis, we identified a significant positive correlation between relative cortical expansion in humans versus chimpanzees, and the gradient of regional prevalence of synergy previously derived from functional MRI ( $\rho=0.42, p=0.001$; Figure 3D). Thus, these findings suggest that the additional cortical tissue gained through human evolution is primarily dedicated to synergy, rather than redundancy.

To provide further support for the evolutionary relevance of synergistic interactions, we capitalised on human adult brain microarray datasets across 57 regions of the left cortical mantle ${ }^{20}$, made available by the Allen Institute for Brain Science (AIBS) ${ }^{21}$. We demonstrate that regional dominance of synergy correlates with regional expression of genes that are both (i) related to brain development and function, including intelligence and synaptic transmission 20; and (ii) selectively accelerated in humans versus non-human primates ("HAR-Brain genes"; $\rho=0.40, p=0.002$; Figure $3 \mathrm{E}$ ). Thus, the more important a brain region is in terms of synergy, the more likely it is to express brain genes that are uniquely human.

Taken together, these findings provide converging evidence for the hypothesis that evolutionary pressures selectively potentiated the role of synergistic interactions in the human brain, both in terms of dedicated genes, (Fig. 3E) dedicated cortical real estate (Fig. 3D), and

202 the end result: higher prevalence of synergy in human brains than non-human primates (Fig. $2033 \mathrm{~A}, \mathrm{~B})$. 
(A)

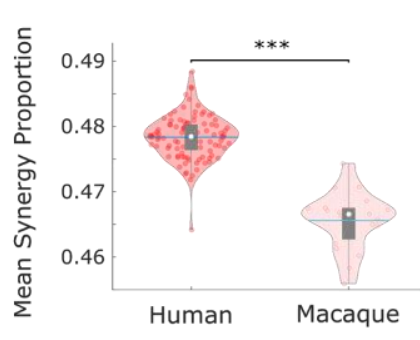

(C)

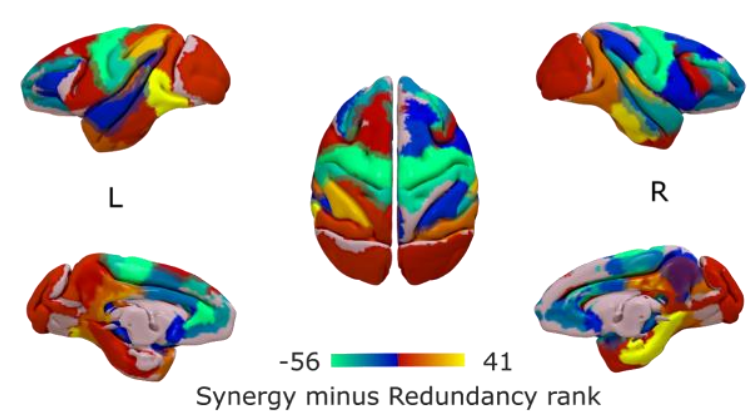

(B)
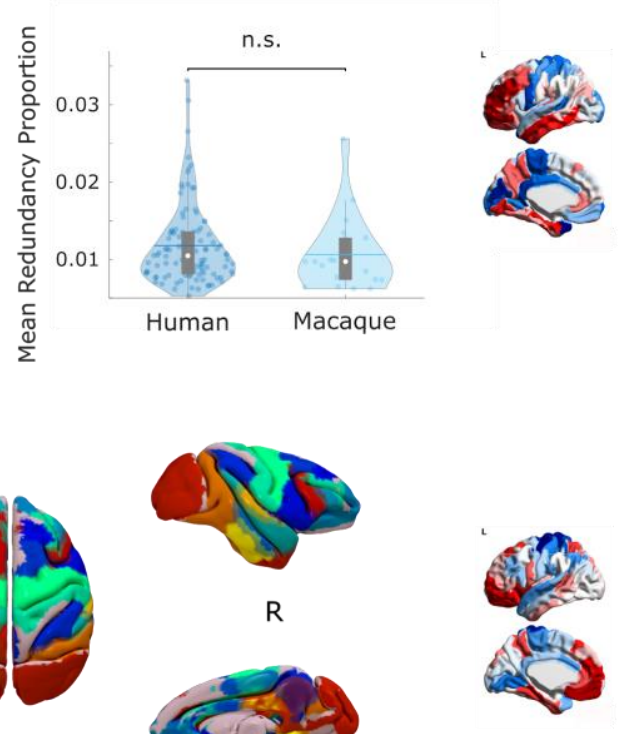

(D)

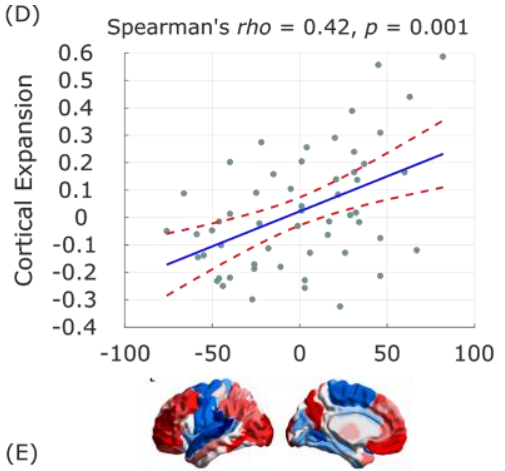

(E)

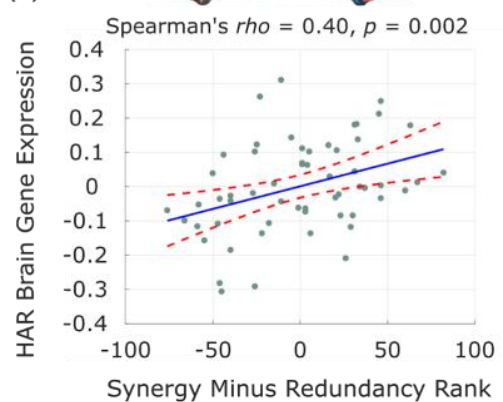

\section{Neurobiological origins of synergy in the human brain}

Figure 3. Human brain evolution favoured high synergy. (A) The proportion of synergistic information exchange across the brain is significantly higher in humans (Homo sapiens) than macaques (Macaca mulatta). (B) The proportion of redundant information exchange across the brain is equivalent in humans and macaques. (C) Surface projection of regional redundancy-to-synergy gradient scores for the macaque brain. (D) Significant correlation between human regional redundancy-to-synergy gradient scores and regional cortical expansion from chimpanzee (Pan troglodytes) to human (both on left hemisphere of DK-114 cortical atlas). (E) Significant correlation between human regional redundancy-to-synergy gradient scores and regional expression of brainrelated human-accelerated (HAR-Brain) genes (both on left hemisphere of DK-114 atlas). The results in (A) and (B) cannot be solely attributed to either the choice of bandpass filter, or the difference in TR between datasets (Figures S9-10). The results in (D) and (E) are also replicated using unadjusted scores (Figure S11).

These observations raise the question of how such high synergy in the human brain could have been attained. To address this question from a neurobiological perspective, we explored the association between the redundancy-to-synergy gradient and regional expression profiles of 20,674 genes from AIBS microarray data ${ }^{10,22}$. Using partial least squares (PLS) regression, we show that the first two PLS components explained $31 \%$ of the variance in the regional synergyredundancy values (Figure S12): significantly more than could be expected by chance (permutation test, $\mathrm{p}=0.007$ ). For both components, gene expression weights were positively correlated with the redundancy-to-synergy regional gradient (PLS1: $\rho=0.37, \mathrm{p}<0.001$; PLS2: $\rho=0.39, \mathrm{p}<0.001$; Figure 4A and Figure S13). These correlations indicate that a number of 
genes are overexpressed in regions where synergy dominates over redundancy -- including significant overexpression of HAR-Brain genes, in line with the results presented above (PLS1: $\mathrm{p}=0.022 ;$ PLS2: $\mathrm{p}<0.001 ;$ Figure S14).

We next sought to identify the role played by overexpressed genes related to brain synergy, for each PLS component. Analysis of gene ontology revealed that the transcriptional signature of PLS2 was significantly enriched in genes involved in learning or memory (in line with our meta-analytic results from NeuroSynth), as well as synapses, synapse components and synaptic transmission (all $\mathrm{p}<10^{-4}$ for significant enrichment).

(A)
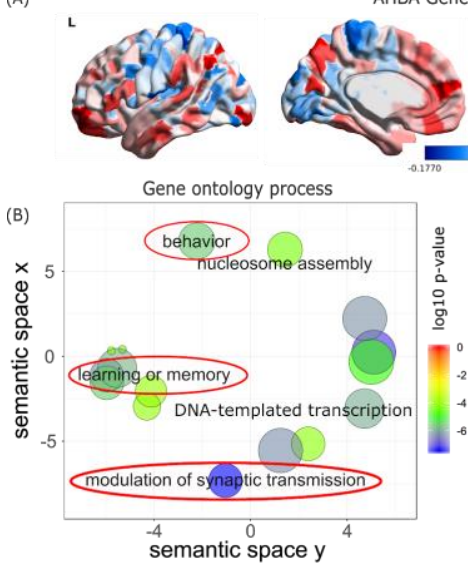

AHBA Genes PLS2 scores

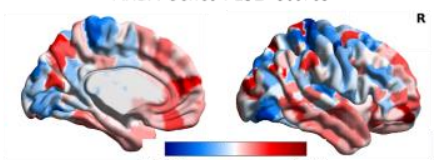

Gene ontology component

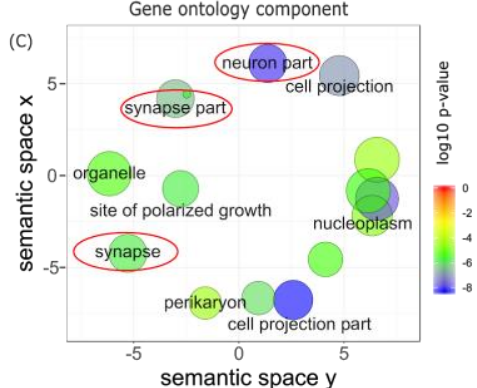

(D)

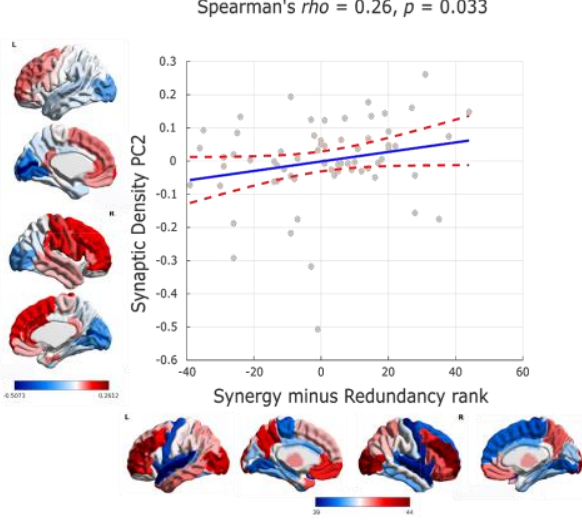

Figure 4. Neurobiological underpinnings of synergy in the human brain. (A) Second principal component of PLS (PLS2) relating the redundancy-to-synergy regional gradient to 20,647 genes from the Allen Institute for Brain Science, for the 308-ROI subdivision of the Desikan-Killiany cortical parcellation. (B) Dimensionalityreduced gene ontology terms pertaining to biological processes that are significantly enriched in PLS2 (red ovals highlight psychologically- or neurobiologically-relevant terms). (C) Dimensionality-reduced gene ontology terms pertaining to cellular components that are significantly enriched in PLS2 (red ovals highlight psychologically- or neurobiologically-relevant terms). Note that semantic space axes indicate the relative distance between terms in multi-dimensional space, but have no intrinsic meaning. Corresponding gene ontology terms for PLS1 are shown in Figure S15. (D) Significant correlation between regional redundancy-to-synergy gradient scores and an anterior-posterior principal component of synaptic density from $\left[{ }^{11} \mathrm{C}\right] \mathrm{UCB}-\mathrm{J}$ PET, for the DK-66 cortical parcellation. Corresponding results for the first principal component of $\left[{ }^{11} \mathrm{C}\right] \mathrm{UCB}-\mathrm{J}$ binding potential are shown in Figure S16.

Synapses are the key structures by which neurons exchange information; therefore they constitute a prime candidate for the neurobiological underpinning of synergistic interactions in the human brain, as suggested by our genetic analysis. To provide a more direct link between synaptic density and regional prevalence of synergy, we used positron emission tomography (PET) to estimate in vivo regional synaptic density based on the binding potential of the 
synapse-specific radioligand $\left[{ }^{11} \mathrm{C}\right] \mathrm{UCB}-\mathrm{J}{ }^{23}$. This radioligand has high affinity for the synaptic vesicle glycoprotein $2 \mathrm{~A}(\mathrm{SV} 2 \mathrm{~A})^{24}$, which is ubiquitously expressed in all synapses throughout the brain ${ }^{25}$. Supporting the notion that regional brain synergy is related to underlying synaptic density, we found that an anterior-posterior principal component of synaptic density derived from $\left[{ }^{11} \mathrm{C}\right] \mathrm{UCB}-\mathrm{J}$ PET is significantly correlated with the regional gradient from redundancy to synergy $(\rho=0.26, p=0.033 ;$ Figure 4D).

Therefore, genetic and molecular evidence converge to indicate synapses and synaptic transmission as key neurobiological underpinnings of synergy in the brain - in line with the notion that synergy quantifies information integration, and its role in supporting higher cognition.

Decomposing interactions between brain regions into synergistic and redundant components illuminates how the brain addresses the inherent trade-off between robustness and integration, providing powerful insights that are beyond traditional methods of studying brain interactions (e.g. FC). Having demonstrated the crucial role of synergistic interactions in human cognitive architecture via meta-analytic and graph-theoretical approaches, we proceeded to identify their neurobiological underpinnings by combining genetic, molecular and neuroanatomical evidence.

Taken together, our findings reveal that basic sensorimotor functions are supported by a modular backbone of redundant interactions (Fig 1D, 2B). As the brain's input-output systems, reliable sensorimotor channels are vital for survival, warranting the additional robustness provided by redundant interactions - as indicated by our structural-functional analysis (Fig. 2D). In contrast, synergistic interactions are ideally poised to act as a global workspace, allowing the integration of complementary information from across the brain in the service of higher cognitive functions (Fig 1D): they bridge across different modules (Fig 1C), form a globally efficient network (Fig 2A), and their neuroanatomical organisation coincides with synapse-rich association cortex (Fig 4D and Supplementary Fig 3).

We further discovered that synergistic interactions were specifically enhanced in humans as a result of evolutionary pressures, with dedicated cortical real estate and dedicated genes, including those promoting synaptic transmission. This process resulted in a neural architecture that is capable of leveraging synergistic information to a greater extent than other primates. Our findings suggest that regions of the default mode and executive control (sub)networks may 
be able to support human higher cognition precisely thanks to their extensive involvement with synergistic processing.

Intriguingly, the high-synergy DMN is involved in self-related cognitive processes ${ }^{26,27}$, and it is also especially disrupted by loss of consciousness, whether caused by anaesthesia or severe brain injury ${ }^{28}$. Indeed, the global workspace theory of consciousness posits that integration of information within a global workspace is necessary for consciousness ${ }^{29}$ - and a formal link has also been established between synergy and the measure of consciousness known as integrated information ${ }^{3,30}$. Therefore, decomposition of information exchange into synergy and redundancy may also shed light on the emergence of consciousness in the human brain providing a framework to discover the information-processing principles that govern how mental phenomena emerge from neurobiology.

Shannon's Mutual information (MI) quantifies the interdependence between two random variables $X$ and $Y$. It is calculated as

$\mathrm{I}(X ; Y)=\mathrm{H}(X)-\mathrm{H}(X \mid Y)=\mathrm{H}(X)+\mathrm{H}(Y)-\mathrm{H}(X, Y)$,

where $H(X)$ stands for the Shannon entropy of a variable $X$. Above, the first equality states that the mutual information is equal to the reduction in entropy (i.e. uncertainty) about $X$ after $Y$

310 becomes accessible. Put simply, the mutual information quantifies the information that one 311 variable provides about another ${ }^{31}$. 
312 Crucially, Williams and Beer (2010) ${ }^{2}$ observed that the information that two source variables

$313 X$ and $Y$ give about a third target variable $Z, \mathrm{I}(X, Y ; Z)$, should be decomposable in terms of

314 different types of information: information provided by one source but not the other (unique

315 information), or by both sources separately (redundant information), or jointly by their

316 combination (synergistic information). Following this intuition, they developed the Partial

317 Information Decomposition (PID ${ }^{2}$ ) framework, which leads to the following fundamental

318 decomposition:

$319 \mathrm{I}(X, Y ; Z)=\operatorname{Red}(X, Y ; Z)+\mathrm{Un}(X ; Z \mid Y)+\mathrm{Un}(Y ; Z \mid X)+\operatorname{Syn}(X, Y ; Z)$.

320 Above, Un corresponds to the unique information one source but the other doesn't, Red is the redundancy between both sources, and $S y n$ is their synergy: information that neither $X$ nor $Y$ alone can provide, but that can be obtained by considering $X$ and $Y$ together. It is worth noticing that the unique information is fully determined after synergistic and redundant comments have been accounted for; hence, we focus our analyses on the two latter components.

The simplest example of a purely synergistic system is one in which $X$ and $Y$ are independent fair coins, and $Z$ is determined by the exclusive-OR function $Z=X \operatorname{OR}(X, Y)$ : i.e., $Z=0$ whenever $X$ and $Y$ have the same value, and $Z=1$ otherwise. It can be shown that $X$ and $Y$ are both statistically independent of $Z$, which implies that neither of them provide - by themselves - information about $Z$. However, $X$ and $Y$ together fully determine $Z$ : hence, the relationship between $Z$ with $X$ and $Y$ is purely synergistic.

While PID provides a formal framework, it does not enforce how the corresponding parts ought to be calculated. While there is ongoing research on the advantages of different decompositions for discrete data, most decompositions converge into the same simple form for the case of continuous Gaussian variables ${ }^{32}$. Known as minimum mutual information PID (MMI-PID), this decomposition quantifies redundancy in terms of the minimum mutual information of each individual source with the target; synergy, then, becomes identified with the additional information provided by the weaker source once the stronger source is known. Since linearGaussian models are sufficiently good descriptors of functional MRI timeseries (and more complex, non-linear models offer no advantage ${ }^{33}$ ), here we adopt the MMI-PID decomposition, following previous applications of PID to neuroscientific data ${ }^{34}$.

341 In a dynamical system such as the brain, one can calculate the amount of information flowing 342 from the system's past to its future, known as time-delayed mutual information 
343 (TDMI). Specifically, by denoting the past of variables as $X_{t-\tau}$ and $Y_{t-\tau}$ and treating them as sources, and their joint future state $\left(X_{t}, Y_{t}\right)$, as target, one can apply the PID framework and decompose the information flowing from past to future as

346

347

348

349

350

351

352

353

354

355

356

357

358

359

360

361

362

363

364

365

$$
\begin{aligned}
I\left(X_{t-\tau}, Y_{t-\tau} ;\right. & \left.X_{t}, Y_{t}\right) \\
& =\operatorname{Red}\left(X_{t-\tau}, Y_{t-\tau} ; X_{t}, Y_{t}\right)+\operatorname{Un}\left(X_{t-\tau} ; X_{t}, Y_{t} \mid Y_{t-\tau}\right) \\
& +\operatorname{Un}\left(Y_{t-\tau} ; X_{t}, Y_{t} \mid X_{t-\tau}\right)+\operatorname{Syn}\left(X_{t-\tau}, Y_{t-\tau} ; X_{t}, Y_{t}\right)
\end{aligned}
$$

Recently, this equation has been refined to also distinguish between redundant, unique, and synergistic information shared with respect to the future variables $X_{t}, Y_{t}$. Importantly, this framework, known as Integrated Information Decomposition (PhiID) ${ }^{3}$, has identified $\operatorname{Syn}\left(X_{t-\tau}, Y_{t-\tau} ; X_{t}, Y_{t}\right)$ with the capacity of the system to exhibit emergent behaviour ${ }^{35}$ [CITE emergence]. Furthermore, PhiID introduced a stronger notion of redundancy, in which information is shared by $X$ and $Y$ in both past and future. Accordingly, using the MMI-PhiID decomposition for Gaussian variables, we use

$\operatorname{Red}(X, Y)=\min \left\{I\left(X_{t-\tau} ; X_{t}\right), I\left(X_{t-\tau} ; Y_{t}\right), I\left(Y_{t-\tau} ; X_{t}\right), I\left(Y_{t-\tau} ; Y_{t}\right)\right\}$

$\operatorname{Syn}(X, Y)=I\left(X_{t-\tau}, Y_{t-\tau} ; X_{t}, Y_{t}\right)-\max \left\{I\left(X_{t-\tau} ; X_{t}, Y_{t}\right), I\left(Y_{t-\tau} ; X_{t}, Y_{t}\right)\right\}$

Here, we used the Gaussian solver implemented in the JIDT toolbox ${ }^{36}$ to obtain TDMI, synergy and redundancy between each pair of brain regions, based on their HRF-deconvolved BOLD signal timeseries (Supplementary Methods).

\section{Gradient of redundancy-to-synergy relative importance}

After building networks of synergistic and redundant interactions between each pair of regions of interest (ROIs), we determined the role of each ROI in terms of its relative engagement in synergistic or redundant interactions. We first calculated the nodal strength of each brain region as the sum of all its connections in the group-averaged matrix. Then, we ranked all 232 regions based on their nodal strength (with higher-strength regions having higher ranks). This 
procedure was done separately for networks of synergy and redundancy. Subtracting each region's redundancy rank from its synergy rank yielded a gradient from negative (i.e. ranking higher in terms of redundancy than synergy) to positive (i.e. having a synergy rank higher than the corresponding redundancy rank); note that the sign is arbitrary.

374 It is important to note that the gradient is based on relative - rather than absolute - differences between regional synergy and redundancy. Consequently, a positive rank difference does not necessarily mean that the region's synergy is greater than its redundancy; rather, it indicates that the balance between its synergy and redundancy relative to the rest of the brain is in favour of synergy - and vice versa for a negative gradient.

The same procedure was also repeated for network edges (instead of nodes), using their weights to rank them separately in terms of synergy and redundancy and then calculating their difference. This produced a single connectivity matrix where each edge's weight represents its relative importance, being higher for synergy (positive edges) or redundancy (negative edges).

\section{NeuroSynth term-based meta-analysis of redundancy-to-synergy gradient}

385

386

The regional redundancy-to-synergy gradient identified in terms of nodal rank differences was related to specific words using NeuroSynth, an online platform for large-scale, automated synthesis of fMRI data [https://neurosynth.org/]. For our analyses we employ 24 topic terms used by previous studies ${ }^{13,16}$, which range from lower sensorimotor functions (such as eye movement, motion, visual and auditory perception) to higher cognitive functions (e.g. attention, working memory, social and numerical cognition).

A meta-analysis analogous to the one implemented by previous studies ${ }^{13,16}$, was conducted to identify topic terms associated with the redundancy-to-synergy gradient. Twenty binary brain masks were obtained by splitting the values of the redundancy-to-synergy gradient into fivepercentile increments. These brain masks served as input for the meta-analysis, based on the chosen 24 topic terms. For visualisation, terms were ordered according to the weighted mean of the resulting Z-statistics. Note that the term "visual semantics" was excluded from visualisation, because it failed to reach the significance threshold of $Z>3.1$, leaving 23 terms (Figure 1). The analyses were carried out using modified code made freely available at [https://www.github.com/gpreti/GSP_StructuralDecouplingIndex]. 
Measures of network integration and segregation

401

402

403

404

405

406

407

408

409

410

411

412

413

414

415

416

417

418

419

420

421

422

423

424

425

426

427

We quantified global integration in the networks of synergistic and redundant connections computing the networks global efficiency, a well-known measure that quantifies the ease of parallel information transfer in the network. More precisely, the global efficiency of a network corresponds to the average of the inverse of the shortest path length between each pair of nodes 37 :

Following Cruzat et al (2018) ${ }^{38}$, segregation of brain networks was quantified by means of network modularity. Put simply, the modularity function quantifies the extent to which a network can be partitioned such that the number of within-group edges is maximised and the density of between-group edges is minimised. We employed an implementation of Newman's spectral modularity algorithm ${ }^{39}$ available in the Brain Connectivity Toolbox (BCT; ${ }^{37,40}$.

$$
G e=\frac{1}{n} \sum_{i}^{n} \frac{\sum_{j \neq i}^{n}\left(d_{i j}\right)^{-1}}{n-1}
$$

\section{Structural-Functional Similarity}

Matrices of synergy and redundancy were thresholded proportionally using the same network density as the structural connectivity matrix of the same subject. This procedure was selected in order to ensure that the same number of edges would be present in both matrices, so that the two matrices can be compared. Then, the upper triangular portion of each connectivity matrix (structural and synergy/redundancy) was flattened into a vector, and the Spearman correlation coefficient between these two vectors was computed. We use this correlation as a measure of similarity between synergy or redundancy and structural connectivity.

\section{HAR-BRAIN genes.}

The maps of regional expression of human-accelerated genes for the DK-114 atlas were made available by Wei et al (2019), where the reader can find detailed information about how these data were generated. Briefly, genes located in a total of 2737 human accelerated regions (HARs) of the genome were taken as presented by comparative genome analysis representing 
genomic loci with accelerated divergence in humans ${ }^{41}$. Out of 2143 HAR-associated genes identified from this procedure, 1711 were described in the Allen Human Brain Atlas (AHBA) microarray dataset (human.brain-map.org) ${ }^{21}$ and were used in the analyses by Wei and colleagues, referred to as HAR genes.

HAR genes were subsequently subdivided into HAR-BRAIN and HAR-NonBRAIN genes. BRAIN genes were selected as the set of genes commonly expressed in human brain tissue using the Genotype-Tissue Expression (GTEx) database (data source: GTEx Analysis Release V6p; https://www.gtexportal.org/), which includes 56,238 gene expression profiles in 53 body sites collected from 7333 postmortem samples in 449 individuals. From these 56,238 genes, a total number of 2823 genes were identified as BRAIN genes showing significantly higher expressions in brain sites than non-brain sites (one-sided t-test and an FDR corrected $\mathrm{q}<0.05$ were used). HAR-BRAIN genes were identified as the 405 genes that overlapped between the 2823 BRAIN genes and the 1711 HAR genes, whereas the remaining HAR genes were labelled as HAR-NonBRAIN genes. Finally, the HAR gene expression data were mapped to the 114region subdivision of the Desikan-Killiany atlas [DK-114] ${ }^{42,43}$. Since only two of the six AHBA donors have data for the right hemisphere, Wei et al (2019) only considered HAR gene expression patterns for the left hemisphere.

\section{Cortical expansion}

447 The maps of evolutionary cortical expansion were made available by Wei et al (2019), ${ }^{20}$ who 448 describe in detail how these data were generated. Briefly, Wei and colleagues analysed in-vivo 449 MRI data from 29 adult chimpanzees, as well as 30 adult human subjects from the Human 450 Connectome Project. Pial surface reconstructions of chimpanzee and human T1-weighted MRI scans (processed with FreeSurefer v5.3.0; https://surfer.nmr.mgh.harvard.edu/) were used for both vertex-to-vertex mapping across chimpanzee and humans and also for subsequent computation of region-wise expansion for cortical morphometry. A regional-level cortical surface area $(\mathrm{Si})$ was computed by summing up face areas within each cortical region, for all regions of the DK-114 atlas ${ }^{42,43}$. Normalized cortical area was obtained by dividing the regional area by the area of the whole cortex. Cortical expansion between every pair of chimpanzee and human subjects was calculated based on both the raw ("unadjusted") and normalized ("adjusted") cortical surface area by 


$$
E_{i, j}=\frac{S_{\text {human }, i}-S_{\text {chimp }, j}}{S_{\text {chimp }, j}}
$$

460 with $\mathrm{E}_{\mathrm{i}, \mathrm{j}}$ denoting the expansion from chimpanzee $\mathrm{j}$ to human $\mathrm{i}$. A group-level region-wise 461 cortical expansion map was calculated by taking averages over the 870 chimpanzee-to-human comparisons.

\section{$464 \quad$ AIBS gene expression analysis}

465 Regional gene expression levels for 20,647 human genes were obtained from transcriptomic 466 measurements in six post-mortem adult brains (age: 24-57 years), made available by the AIBS 467 (human.brain-map.org) ${ }^{21}$. We used code made freely available by Morgan et al (2019) ${ }^{10}$ 468 https://github.com/SarahMorgan/Morphometric_Similarity_SZ) to obtain a 308 x 20,647 regional 469 transcription matrix, matching gene expression data to each cortical region of the DK-308 atlas $47010,22,44,45$ (Supplementary Methods). Each tissue sample was assigned to a cortical region using the 471 AIBS MRI data for each donor, pooling samples between bilaterally homologous regions ${ }^{10,45}$.

\section{Partial Least Squares}

474 To explore the association between the redundancy-to-synergy regional gradient and all 20,647

475 genes measured in the AHBA microarrays, at each of 308 regions, we used partial least squares 476 (PLS) as a dimensionality reduction technique ${ }^{10,22,44,46}$. PLS finds components from the predictor 477 variables ( $308 \times 20,647$ matrix of regional gene expression scores) that have maximum covariance 478 with the response variables $(308 \times 1$ matrix of regional redundancy-to-synergy gradient). The PLS 479 components (i.e. linear combinations of the weighted gene expression scores) are ranked by 480 covariance between predictor and response variables, so that the first few PLS components provide 481 a low-dimensional representation of the covariance between the higher dimensional data matrices.

482 Goodness of fit of low-dimensional PLS components was tested non-parametrically by repeating 483 the analysis 1000 times after shuffling the regional labels. The error on the PLS weights associated 484 with each gene were tested by resampling with replacement of 308 ROIs (bootstrapping); the ratio 485 of the weight of each gene to its bootstrap standard error was used to Z-score the genes and rank 486 their contributions to each PLS component ${ }^{10,22,44}$. 


\section{Gene ontology and enrichment analysis}

489 We used GOrilla for enrichment analysis of the first two PLS components ${ }^{22,47}$ GOrilla identifies 490 enriched gene ontology (GO) terms in ranked gene list, leveraging a large online database of gene 491 annotations corresponding to 'biological processes' and 'cellular components' ${ }^{47}$ We identified GO 492 terms that were over-represented among the genes with the strongest positive weightings on each 493 PLS component (i.e. those most strongly associated with dominance of synergy over redundancy). 494 For our analyses on the online GOrilla platform (http://cbl-gorilla.cs.technion.ac.il) we unchecked 495 the "Run GOrilla in fast mode" option and used the "P-value threshold 10-4" setting in order to 496 best approximate FDR correction with $\alpha=0.05^{22}$.

497 We then used the online tool REViGO (http://revigo.irb.hr) to summarize the list of significant GO 498 terms and visualize the results of whole-genome enrichment analysis. First, REViGO employs 499 measures of semantic similarity between terms ${ }^{48}$ to identify representative clusters of genes. Then, 500 REViGO plots significant GO terms in semantic space, where semantically similar GO terms are 501 represented clustered near one another and labelled in a representative manner.

502 For our hypothesis-driven analysis, testing for enrichment of HAR-Brain genes, we also used non503 parametric permutation testing. Specifically, we randomly drew 1000 samples of the same number 504 of genes and estimated their PLS weighting, and compared the PLS weights of the HAR-Brain 505 genes to this permutation distribution. This provided an estimate of the probability of HAR-Brain 506 gene enrichment of each PLS component under the null hypothesis ${ }^{10,22}$. We note that this 507 permutation procedure does not take into account the correlation between HAR-Brain genes; more 508 sophisticated null models for permutation testing that controlled for these or other characteristics 509 of candidate genes will be important to develop for computational inference in future studies.

\section{Synaptic Density from Positron Emission Tomography}

513 In-vivo estimates of regional synaptic density in the human brain were obtained from positron 514 emission tomography (PET) with the radioligand $\left[{ }^{11} \mathrm{C}\right] \mathrm{UCB}-\mathrm{J}\left((\mathrm{R})-1-\left(\left(3-\left(\right.\right.\right.\right.$ methyl- $\left.{ }^{11} \mathrm{C}\right)$ pyridin-

515 4-yl)methyl)-4- (3,4,5-trifluorophenyl)pyr-rolidin-2-one) ${ }^{49}$. This ligand quantifies synaptic 
516 density ${ }^{23}$ based on its affinity for the presynaptic vesicle glycoprotein $2 \mathrm{~A}(\mathrm{SV} 2 \mathrm{~A}){ }^{24}$ which is

517 ubiquitously expressed in all brain synapses ${ }^{25}$.

519 PET/MR imaging protocol

520 The research protocol was approved by an NHS Research Ethics Committee (REC: 521 18/EE/0059) and the Administration of Radioactive Substances Advisory Committee 522 (ARSAC), and all participants provided written informed consent in accordance with the 523 Declaration of Helsinki. Participant recruitment and exclusion criteria are described in detail 524 in the original publication ${ }^{49}$. Here, we included data from the healthy volunteers $(\mathrm{N}=15,8$ 525 females; age: $68 \pm 7$ years).

526 The radioligand $\left[{ }^{11} \mathrm{C}\right] \mathrm{UCB}-\mathrm{J}$ was synthesised at the Radiopharmacy Unit, Wolfson Brain 527 Imaging Centre, Cambridge University, using the methodology previously described ${ }^{24}$. All 528 participants underwent simultaneous 3T MRI and $\left[{ }^{11} \mathrm{C}\right] \mathrm{UCB}-\mathrm{J}$ PET on a GE SIGNA PET/MR 529 (GE Healthcare, Waukesha, USA). Dynamic PET data acquisition was performed for 90 530 minutes starting immediately after [ $\left[{ }^{11} \mathrm{C}\right] \mathrm{UCB}-\mathrm{J}$ injection (median (range) injected activity: 408 531 (192-523) MBq, injected UCB-J mass $\leq 10 \mu \mathrm{g}$ ). Attenuation correction included the use of a 532 multi-subject atlas method ${ }^{50}$ and improvements to the MRI brain coil component ${ }^{51}$. Each emission image series was aligned using SPM12 (www.fil.ion.ucl.ac.uk/spm/software/spm12/)

534 then rigidly registered to a T1-weighted MRI acquired during PET data acquisition (TR = 3.6 $535 \mathrm{msec}, \mathrm{TE}=9.2 \mathrm{msec}, 192$ sagittal slices, in plane resolution $0.55 \times 0.55 \mathrm{~mm}$ (subsequently 536 interpolated to $1.0 \times 1.0 \mathrm{~mm}$ ); slice thickness $1.0 \mathrm{~mm}$ ). Regional time-activity curves were 537 extracted following the application of geometric transfer matrix partial volume correction ${ }^{51}$ to 538 each of the dynamic PET images. To quantify SV2A density (and therefore synaptic density), 539 regional $\left[{ }^{11} \mathrm{C}\right] \mathrm{UCB}-\mathrm{J}$ non-displaceable binding potential $\left(\mathrm{BP}_{\mathrm{ND}}\right)$ was determined for a $66-\mathrm{ROI}$ 540 subdivision of the Desikan-Killiany cortical atlas (DK-66), using a basis function

541 implementation of the simplified reference tissue model ${ }^{52}$, with the reference tissue defined in 542 the centrum semiovale ${ }^{53,54}$. 


\section{Principal components of synaptic density}

545 Principal Components Analysis (PCA) was subsequently employed to derive the principal

546 components that explain most of the variance in regional $\left[{ }^{11} \mathrm{C}\right] \mathrm{UCB}-\mathrm{J}$ BP $\mathrm{ND}$ across volunteers.

547 Components were selected if their associated eigenvalue was greater than unity; two principal 548 components satisfied this criterion, explaining $45 \%$ and $16 \%$ of the variance, respectively.

\section{Acknowledgements}

552 Funding

553 This work was supported by grants from the National Institute for Health Research (NIHR,

554 UK), Cambridge Biomedical Research Centre and NIHR Senior Investigator Awards [to

555 DKM]; the British Oxygen Professorship of the Royal College of Anaesthetists [to DKM]; the

556 Stephen Erskine Fellowship (Queens' College, Cambridge), [to EAS]; and the Gates

557 Cambridge Trust (to AIL). PAM and DB are funded by the Wellcome Trust (grant no.

558 210920/Z/18/Z). FR is funded by the Ad Astra Chandaria foundation. Computing

559 infrastructure at the Wolfson Brain Imaging Centre (WBIC-HPHI) was funded by the

560 MRC research infrastructure award (MR/M009041/1).

561 The PET study was funded by the Cambridge University Centre for Parkinson-Plus; the

562 National Institute for Health Research Cambridge Biomedical Research Centre (146281); the

563 Wellcome Trust (103838) and the Association of British Neurologists, Patrick Berthoud

564 Charitable Trust (RG99368).

565 Data were provided [in part] by the Human Connectome Project, WU-Minn Consortium

566 (Principal Investigators: David Van Essen and Kamil Ugurbil; 1U54MH091657) funded by the

56716 NIH Institutes and Centers that support the NIH Blueprint for Neuroscience Research; and

568 by the McDonnell Center for Systems Neuroscience at Washington University. 
569 For the macaque data, primary support for the work by Newcastle University was provided by

570 Wellcome Trust (WT091681MA, WT092606AIA), National Centre for 3Rs (Project grant

571 NC/K000802/1; Pilot grant NC/K000608/1), and BBSRC (grant number BB/J009849/1).

572 We express our gratitude to the Primate neuroimaging Data- Exchange (PRIME-DE) initiative,

573 to the organizers and managers of PRIME-DE and to all the institutions that contributed to the

574 PRIME-DE dataset (http://fcon_1000.projects.nitrc.org/indi/indiPRIME.html), with special

575 thanks to the Newcastle team. We are also grateful to Anoine Grigis, Jordy Tasserie and Bechir

576 Jarraya for their help with the Pypreclin code, and Rodrigo Romero-Garcia for generating and

577 sharing the $500 \mathrm{~mm} 2$ subparcellation of the DK atlas, and the corresponding Von Economo

578 cytoarchitectonics map. We are also grateful to Yongbin Wei and colleagues for generating

579 and making available the data pertaining to HAR genes and cortical expansion. We are grateful

580 to UCB Pharma for providing the precursor for the radioligand used in PET imaging.

581

582

\section{Author Contributions}

583

AIL: conceived the study; analysed data; wrote first draft of the manuscript. PAM: conceived

584

the study; contributed to data analysis; reviewed and edited the manuscript. FR: contributed to

585 data analysis; reviewed and edited the manuscript. NH: acquired PET data; reviewed PET

586 analysis; reviewed the manuscript. TDF: preprocessed PET data; reviewed the manuscript.

587 JOB: conceived the PET project; reviewed PET analysis; reviewed the manuscript. JBR: conceived the PET project; reviewed PET analysis; reviewed the manuscript. DKM: reviewed the manuscript. DB: conceived the study; reviewed and edited the manuscript. EAS: conceived

590 the study; reviewed and edited the manuscript.

\section{Competing Interests}

JBR serves as an associate editor to Brain, and is a non-remunerated trustee of the Guarantors 594 of Brain and the PSP Association (UK). He provides consultancy to Asceneuron, Biogen and

595 UCB and has research grants from AZ-Medimmune, Janssen and Lilly as industry partners in the Dementias Platform UK. All other authors declare no conflicts of interest. 
599 The HCP DWI data in SRC format are available online

600 (http://brain.labsolver.org/diffusion-mri-data/hcp-dmri-data). The HCP fMRI data are

601 available online (https://www.humanconnectome.org/study/hcp-young-adult/data-releases).

602

603 Macaque MRI data are available from the PRIMatE Data Exchange (PRIME-DE) through the

604 Neuroimaging Informatics Tools and Resources Clearinghouse (NITRC;

605 http://fcon_1000.projects.nitrc. org/indi/indiPRIME.html).

606

607 The PET data that support the findings of this study are available from author NH

608 (nda26@medschl.cam.ac.uk), upon reasonable request for academic (non-commercial)

609 purposes.

610

611 The macaque connectome is available online on Zenodo:

612 https://zenodo.org/record/1471588\#.X2JCjdZuJPY

613

614 Cortical gene expression patterns were taken from the transcriptomic data of the Allen Human

615 Brain Atlas (AHBA, http://human.brain- map.org/static/download).

616 Region-wise maps of chimpanzee-to-human cortical expansion and HAR gene expression are

617 available as Supplementary Materials from Wei et al (2019) ${ }^{20}$.

618 The NMT anatomical volume and associated probabilistic tissue segmentation maps (GM,

619 WM and CSF) are freely available online: https://afni.nimh.nih.gov/pub/

620 dist/atlases/macaque/nmt and http://github.com/jms290/NMT. 
626 The Java Information Dynamics Toolbox is freely available online:

627 (https://github.com/jlizier/jidt).

628 The CONN toolbox is freely available online (http://www.nitrc.org/projects/conn).

629 DSI Studio is freely available online (www.dsi-studio.labsolver.org).

630 The Brain Connectivity Toolbox code used for graph-theoretical analyses is freely available

631 online (https://sites.google.com/site/bctnet/).

632 The code used for NeuroSynth meta-analysis is freely available online:

633 (https://www.github.com/gpreti/GSP_StructuralDecouplingIndex).

634 The HRF deconvolution toolbox is freely available online:

635 (https://www.nitrc.org/projects/rshrf).

636 The Pypreclin pipeline code is freely available at GitHub

637 (https://github.com/neurospin/pypreclin).

638 The code for PLS analysis of gene expression profiles is freely available online:

639 https://github.com/SarahMorgan/Morphometric_Similarity_SZ.

\section{References}

642 1. Marr, D. Vision : a computational investigation into the human representation and

643 processing of visual information. (MIT Press, 2010).

6442 Williams, P. L. \& Beer, R. D. Nonnegative Decomposition of Multivariate Information.

$645 \quad$ (2010).

646 3. Mediano, P. A. M., Rosas, F., Carhart-Harris, R. L., Seth, A. K. \& Barrett, A. B.

647 Beyond integrated information: A taxonomy of information dynamics phenomena.

$648 \quad$ arXiv (2019). 
649 4. Whitacre, J. M. Biological robustness: Paradigms, mechanisms, systems principles.

5. Tononi, G., Sporns, O. \& Edelman, G. M. A measure for brain complexity: relating functional segregation and integration in the nervous system. Proc. Natl. Acad. Sci. 91, 5033-5037 (1994).

6. Latham, P. E. \& Nirenberg, S. Synergy, redundancy, and independence in population codes, revisited. J. Neurosci. 25, 5195-5206 (2005).

7. Schneidman, E., Still, S., Berry, M. J. \& Bialek, W. Network information and connected correlations. Phys. Rev. Lett. 91, (2003).

8. Rosas, F. E., Mediano, P. A. M., Gastpar, M. \& Jensen, H. J. Quantifying high-order interdependencies via multivariate extensions of the mutual information. Phys. Rev. $E$ 100, 32305 (2019).

9. Wibral, M., Priesemann, V., Kay, J. W., Lizier, J. T. \& Phillips, W. A. Partial information decomposition as a unified approach to the specification of neural goal functions. Brain Cogn. 112, 25-38 (2017).

10. Morgan, S. E. et al. Cortical patterning of abnormal morphometric similarity in psychosis is associated with brain expression of schizophrenia-related genes. Proc. Natl. Acad. Sci. U. S. A. 116, 9604-9609 (2019).

11. Yeo, B. T. T. et al. The organization of the human cerebral cortex estimated by intrinsic functional connectivity. J. Neurophysiol. 106, 1125-1165 (2011).

12. Rosas, F., Mediano, P., Rassouli, B. \& Barrett, A. An operational information decomposition via synergistic disclosure. (2020).

13. Margulies, D. S. et al. Situating the default-mode network along a principal gradient of macroscale cortical organization. Proc. Natl. Acad. Sci. U. S. A. 113, 12574-12579 (2016).

14. Buckner, R. L. \& Krienen, F. M. The evolution of distributed association networks in the human brain. Trends Cogn. Sci. 17, 648-665 (2013).

15. Jones, E. G. \& Powell, T. P. S. An anatomical study of converging sensory pathways within the cerebral cortex of the monkey. Brain 93, 793-820 (1970).

16. Preti, M. G. \& Van De Ville, D. Decoupling of brain function from structure reveals regional behavioral specialization in humans. Nat. Commun. 10, (2019).

17. Tononi, G. \& Edelman, G. M. Consciousness and Complexity.

18. Sporns, O. Networks of the brain. (MIT Press, 2011).

19. Smaers, J. B., Gómez-Robles, A., Parks, A. N. \& Sherwood, C. C. Exceptional Evolutionary Expansion of Prefrontal Cortex in Great Apes and Humans. Curr. Biol. 27, 714-720 (2017).

20. Wei, Y. et al. Genetic mapping and evolutionary analysis of human-expanded cognitive networks. Nat. Commun. 10, (2019).

21. Hawrylycz, M. J. et al. An anatomically comprehensive atlas of the adult human brain transcriptome. Nature 489, 391-399 (2012).

22. Whitaker, K. J. et al. Adolescence is associated with genomically patterned consolidation of the hubs of the human brain connectome. Proc. Natl. Acad. Sci. U. S. A. 113, 9105-9110 (2016).

23. Finnema, S. J. et al. Imaging synaptic density in the living human brain. Sci. Transl. Med. 8, (2016).

24. Milicevic Sephton, S. et al. Automated radiosynthesis of [11C]UCB-J for imaging synaptic density by positron emission tomography. J. Label. Compd. Radiopharm. 63, 151-158 (2020).

25. Bajjalieh, S. M., Frantz, G. D., Weimann, J. M., McConnell, S. K. \& Scheller, R. H. Differential expression of synaptic vesicle protein 2 (SV2) isoforms. J. Neurosci. 14, 5223-5235 (1994).

26. Cavanna, A. E. \& Trimble, M. R. The precuneus: A review of its functional anatomy and behavioural correlates. Brain 129, 564-583 (2006).

27. Qin, P. \& Northoff, G. How is our self related to midline regions and the default-mode network? Neuroimage 57, 1221-1233 (2011). 
28. Luppi, A. I. et al. Consciousness-specific dynamic interactions of brain integration and functional diversity. Nat. Commun. (2019).

29. Mashour, G. A., Roelfsema, P., Changeux, J. P. \& Dehaene, S. Conscious Processing and the Global Neuronal Workspace Hypothesis. Neuron 105, 776-798 (2020).

30. Griffith, V. A Principled Infotheoretic phi-like Measure. bioRxiv (2014).

31. Cover, T. M. \& Thomas, J. A. Elements of Information Theory. Elements of Information Theory (Wiley-Interscience, 2005). doi:10.1002/047174882X

32. Barrett, A. B. Exploration of synergistic and redundant information sharing in static and dynamical Gaussian systems. Phys. Rev. E 91, 52802 (2015).

33. Schulz, M.-A. et al. Deep learning for brains?: Different linear and nonlinear scaling in UK Biobank brain images vs. machine-learning datasets. bioRxiv 5, 16 (2019).

34. Bím, J. et al. A Non-negative Measure Of Feature-Related Information Transfer Between Neural Signals. bioRxiv doi: https, (2019).

35. Rosas, F. E. et al. Reconciling emergences: An information-theoretic approach to identify causal emergence in multivariate data.

36. Lizier, J. T. JIDT: An Information-Theoretic Toolkit for Studying the Dynamics of Complex Systems. Front. Robot. Al 1, 1-37 (2014).

37. Rubinov, M. \& Sporns, O. Complex network measures of brain connectivity: Uses and interpretations. Neuroimage 52, 1059-1069 (2010).

38. Cruzat, J. et al. The dynamics of human cognition: Increasing global integration coupled with decreasing segregation found using iEEG. Neuroimage 172, 492-505 (2018).

39. Newman, M. E. J. Modularity and community structure in networks. Proc. Natl. Acad. Sci. U. S. A. 103, 8577-8582 (2006).

40. Rubinov, M. \& Sporns, O. Weight-conserving characterization of complex functional brain networks. Neuroimage 56, 2068-2079 (2011).

41. Doan, R. N. et al. Mutations in Human Accelerated Regions (HARs) Disrupt Cognition and Social Behavior The Homozygosity Mapping Consortium for Autism HHS Public Access. Cell 167, 341-354 (2016).

42. Desikan, R. S. et al. An automated labeling system for subdividing the human cerebral cortex on MRI scans into gyral based regions of interest. Neuroimage 31, 968-980 (2006).

43. Cammoun, L. et al. Mapping the human connectome at multiple scales with diffusion spectrum MRI. J. Neurosci. Methods 203, 386-397 (2012).

44. Vértes, P. E. et al. Gene transcription profiles associated with inter-modular hubs and connection distance in human functional magnetic resonance imaging networks.

Philos. Trans. R. Soc. B Biol. Sci. 371, (2016).

45. Romero-Garcia, R. et al. Structural covariance networks are coupled to expression of genes enriched in supragranular layers of the human cortex. Neuroimage 171, 256267 (2018).

46. Krishnan, A., Williams, L. J., Mclntosh, A. R. \& Abdi, H. Partial Least Squares (PLS) methods for neuroimaging: A tutorial and review. Neuroimage 56, 455-475 (2011).

47. Eden, E., Navon, R., Steinfeld, I., Lipson, D. \& Yakhini, Z. GOrilla: A tool for discovery and visualization of enriched GO terms in ranked gene lists. BMC Bioinformatics 10, (2009).

48. Supek, F., Bošnjak, M., `Kunca, S. \& `Muc, S. Summarizes and Visualizes Long Lists of Gene Ontology Terms. PLoS One 6, 21800 (2011).

49. Holland, N. et al. Synaptic Loss in Primary Tauopathies Revealed by [11C]UCB-J Positron Emission Tomography. Mov. Disord. (2020). doi:10.1002/mds.28188

50. Burgos, N. et al. Attenuation correction synthesis for hybrid PET-MR scanners: application to brain studies. IEEE Trans. Med. Imaging 33, 2332-41 (2014).

51. Manavaki, R., Hong, Y. \& Fryer, T. D. Effect of Brain MRI Coil Attenuation Map Processing on PET Image Quantification and Uniformity for the GE SIGNA PET / MR. IEEE Nucl Sci Symp Med Imaging Conf Proc. 30 (2019). 
bioRxiv preprint doi: https:/doi org/10.1101/2020.09.22 308981; this version posted September 22,2020 . The copyright holder for this

preprint (which was not certified by peer review) is the author/funder, who has granted bioRxiv a license to display the preprint in perpetuity. It is made available under aCC-BY 4.0 International license.

52. Wu, Y. \& Carson, R. E. Noise Reduction in the Simplified Reference Tissue Model for Neuroreceptor Functional Imaging. J. Cereb. Blood Flow Metab. 22, 1440-1452 (2002).

53. Koole, M. et al. Quantifying SV2A density and drug occupancy in the human brain using [11C]UCB-J PET imaging and subcortical white matter as reference tissue. Eur. J. Nucl. Med. Mol. Imaging 46, 396-406 (2019).

54. Rossano, S. et al. Assessment of a white matter reference region for 11C-UCB-J PET quantification. J. Cereb. Blood Flow Metab. 40, 1890-1901 (2020). 